Acta Crystallographica Section E

Structure Reports

Online

ISSN 1600-5368

\section{Disorder in ondansetron hydrochloride dihydrate}

The structure of the title compound, 2-methyl-1-(1,2,3,9tetrahydro-9-methyl-4-oxo- $4 H$-carbazol-3-ylmethyl)- $1 H$-imidazol-3-ium chloride dihydrate, $\mathrm{C}_{18} \mathrm{H}_{24} \mathrm{~N}_{3}{ }^{+} \cdot \mathrm{Cl}^{-} \cdot 2 \mathrm{H}_{2} \mathrm{O}$, has been reported previously by Collin, Moureau, Quintero, Vercauteren, Evrard \& Durant [(1995). J. Chem. Soc. Perkin Trans. 2, pp. 77-84] and Chandra Mohan \& Ravikumar, [(1995), Acta Cryst. C51, 2627-2629]. In both determinations, all atoms were refined as clearly ordered. In contrast to this, we present here a redetermination of this structure from new intensity data where two atoms of the cyclohexenone ring are disordered over two sites. Apart from this disorder, our results agree with the already published data.

\section{Comment}

The title compound, (I), is an anti-emetic, which has been described by Milne \& Heel (1991). It is a highly selective 5HT3 receptor antagonist (Ye et al., 2001). A perspective view is shown in Fig. 1.

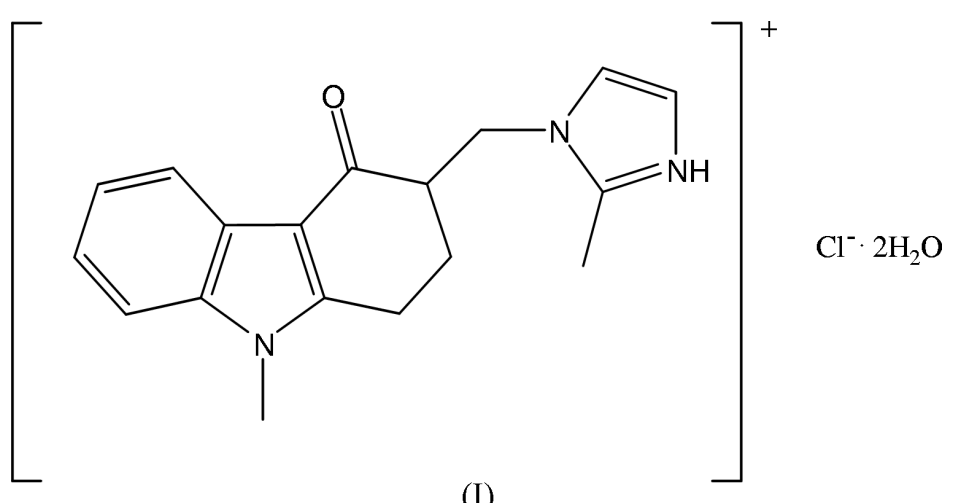

The structure of (I) has already been determined twice at room temperature by two different research groups (Collin et al., 1995; Chandra Mohan \& Ravikumar, 1995). Both of these structures were refined as perfectly ordered. However, we have collected data at low temperature and discovered that two atoms of the cyclohexenone ring are disordered over two sites. In order to check if the crystal had undergone a phase transition upon cooling, a data set was collected on a different crystal at room temperature. This crystal showed the same disorder as the crystal investigated at low temperature. As a result, a phase transition can be ruled out. A closer look at both published ordered structures shows that the displacement ellipsoids of the atoms in question ( $\mathrm{C} 2$ and $\mathrm{C} 3$ in the present structure) are elongated, which suggest disorder. Furthermore, the bond length between these atoms is significantly shortened in the two published structures (1.479 and
Received 3 November 2004 Accepted 9 November 2004 Online 11 December 2004
(C) 2005 International Union of Crystallography Printed in Great Britain - all rights reserved 
$1.485 \AA$ ) compared with the value [1.536 (3) $\mathrm{]}$ ] we found in the major component. This indicates an average of two positions for the two atoms. As a result, the previous structures seem to be disordered as well, but were not refined as such. Apart from this disorder, our results agree with the already published data. Bond lengths and angles can be regarded as normal (Cambridge Structural Database, Version 1.6 plus three updates; Mogul Version 1.0; Allen, 2002).

\section{Experimental}

The sample of the title compound was obtained as a gift from CIPLA, India. It was used without further purification and recrystallized from methanol to give colourless blocks.

\section{Crystal data \\ $\mathrm{C}_{18} \mathrm{H}_{20} \mathrm{~N}_{3} \mathrm{O}^{+} \cdot \mathrm{Cl}^{-} \cdot 2 \mathrm{H}_{2} \mathrm{O}$ \\ $M_{r}=365.85$ \\ Monoclinic, $P 2_{1} / c$ \\ $a=15.0216(13) \AA$ \\ $b=9.6464(8) \AA$ \\ $c=12.6545(10) \AA$ \\ $\beta=100.708(6)^{\circ}$ \\ $V=1801.8(3) \AA^{3}$ \\ $Z=4$}

$$
\begin{aligned}
& D_{x}=1.349 \mathrm{Mg} \mathrm{m}^{-3} \\
& \text { Mo } K \alpha \text { radiation } \\
& \text { Cell parameters from } 20705 \\
& \quad \text { reflections } \\
& \theta=3.7-27.6^{\circ} \\
& \mu=0.23 \mathrm{~mm}^{-1} \\
& T=173(2) \mathrm{K} \\
& \text { Block, colourless } \\
& 0.33 \times 0.27 \times 0.14 \mathrm{~mm}
\end{aligned}
$$

\section{Data collection}

$\begin{array}{ll}\text { Stoe IPDS-II two-circle } & 4181 \text { independent reflections } \\ \quad \text { diffractometer } & 3172 \text { reflections with } I>2 \sigma(I) \\ \omega \text { scans } & R_{\text {int }}=0.058 \\ \text { Absorption correction: multi-scan } & \theta_{\max }=27.7^{\circ} \\ \quad(M U L A B S ; \text { Spek, 1990; Blessing, } & h=-19 \rightarrow 19 \\ \quad 1995) & k=-12 \rightarrow 12 \\ \quad T_{\min }=0.928, T_{\max }=0.967 & l=-16 \rightarrow 16 \\ 24157 \text { measured reflections } & \\ \text { Refinement } & \\ \text { Refinement on } F^{2} & \\ R\left[F^{2}>2 \sigma\left(F^{2}\right)\right]=0.033 & \text { H atoms treated by a mixture of } \\ w R\left(F^{2}\right)=0.087 & \text { independent and constrained } \\ S=0.96 & \text { refinement } \\ 4181 \text { reflections } & w=1 /\left[\sigma^{2}\left(F_{o}^{2}\right)+(0.0567 P)^{2}\right] \\ 267 \text { parameters } & \text { where } P=\left(F_{o}^{2}+2 F_{c}^{2}\right) / 3 \\ & (\Delta / \sigma)_{\max }<0.001 \\ & \Delta \rho_{\max }=0.24 \mathrm{e} \AA^{-3} \\ & \Delta \rho_{\min }=-0.21 \mathrm{e} \AA^{-3}\end{array}$

Table 1

Hydrogen-bonding geometry $\left(\AA{ }^{\circ}\right)$.

\begin{tabular}{lllll}
\hline$D-\mathrm{H} \cdots A$ & $D-\mathrm{H}$ & $\mathrm{H} \cdots A$ & $D \cdots A$ & $D-\mathrm{H} \cdots A$ \\
\hline $\mathrm{N} 23-\mathrm{H} 23 \cdots \mathrm{O} 1 W$ & $0.92(2)$ & $1.75(2)$ & $2.6663(16)$ & $178.0(19)$ \\
$\mathrm{O} 1 W-\mathrm{H} 1 W B \cdots \mathrm{Cl} 1$ & $0.82(2)$ & $2.32(2)$ & $3.1252(14)$ & $166(2)$ \\
$\mathrm{O} 2 W-\mathrm{H} 2 W B \cdots \mathrm{Cl} 1$ & $0.89(3)$ & $2.33(3)$ & $3.2171(15)$ & $175(2)$ \\
$\mathrm{O} W-\mathrm{H} 1 W A \cdots \mathrm{Cl} 1^{\mathrm{i}}$ & $0.85(3)$ & $2.32(3)$ & $3.1334(14)$ & $161(2)$ \\
$\mathrm{O} W-\mathrm{H} 2 W A \cdots{ }^{\mathrm{ii}}$ & $0.82(3)$ & $2.41(3)$ & $3.2205(15)$ & $172(2)$ \\
\hline
\end{tabular}

Symmetry codes: (i) $-x, y-\frac{1}{2}, \frac{1}{2}-z$; (ii) $-x, 1-y,-z$.

The site-occupation factors of the disordered atoms refined to 0.791 (6) and 0.209 (6). In the second crystal examined at room temperature, the ratio of the site-occupation factors refined to $0.759(9) / 0.241(9) . \mathrm{H}$ atoms were refined with fixed individual displacement parameters $\left[U_{\text {iso }}(\mathrm{H})=1.2 U_{\text {eq }}(\mathrm{C})\right.$ or $\left.1.5 U_{\text {eq }}\left(\mathrm{C}_{\text {methyl }}\right)\right]$ using a riding model, with $\mathrm{C}-\mathrm{H}=1.00 \AA, \mathrm{C}-\mathrm{H}=0.99 \AA, \mathrm{C}-\mathrm{H}=$ 0.98 and $0.95 \AA$, for tertiary $\mathrm{CH}$, secondary $\mathrm{CH}$, methyl $\mathrm{CH}$ and

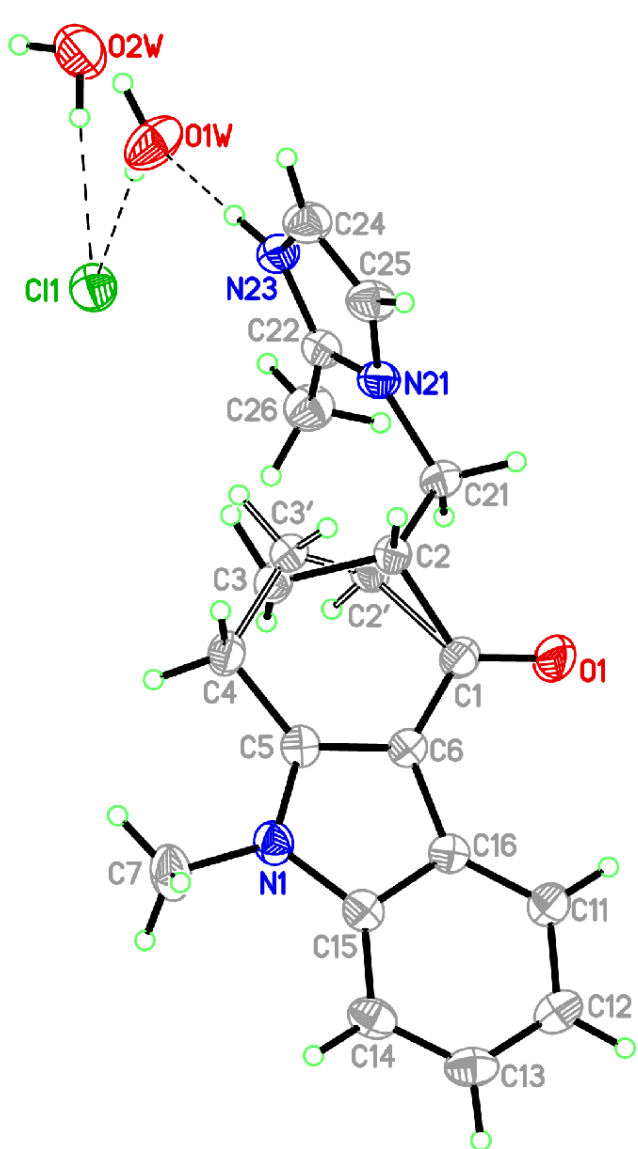

Figure 1

Perspective view of the title compound, with the atom numbering. Displacement ellipsoids are drawn at the $50 \%$ probability level.

aromatic $\mathrm{CH}$, respectively. The methyl groups were allowed to rotate but not to tip. $\mathrm{H}$ atoms bonded to $\mathrm{O}$ and $\mathrm{N}$ atoms were refined isotropically.

Data collection: $X$-AREA (Stoe \& Cie, 2001); cell refinement: $X$-AREA; data reduction: $X$-AREA; program(s) used to solve structure: SHELXS97 (Sheldrick, 1990); program(s) used to refine structure: SHELXL97 (Sheldrick, 1997); molecular graphics: XP in SHELXTL-Plus (Sheldrick, 1991); software used to prepare material for publication: SHELXL97 and PLATON (Spek, 2003).

One of the authors (HSY) thanks CIPLA, Mumbai, for a gift sample of ondansetron hydrochloride dihydrate.

\section{References}

Allen, F. H. (2002). Acta Cryst. B58, 380-388.

Blessing, R. H. (1995). Acta Cryst. A51, 33-38.

Chandra Mohan, K. \& Ravikumar, K. (1995). Acta Cryst. C51, 2627-2629.

Collin, S., Moureau, F., Quintero, M. G., Vercauteren, D. P., Evrard, G. \& Durant, F. (1995). J. Chem. Soc. Perkin Trans. 2, pp. 77-84.

Milne, R. J. \& Heel, R. C. (1991). Drugs, 41, 574-575.

Sheldrick, G. M. (1990). Acta Cryst. A46, 467-473.

Sheldrick, G. M. (1991). SHELXTL-Plus. Release 4.1. Siemens Analytical X-ray Instruments Inc., Madison, Wisconsin, USA.

Sheldrick, G. M. (1997). SHELXL97. University of Göttingen, Germany.

Spek, A. L. (2003). J. Appl. Cryst. 36, 7-12.

Stoe \& Cie (1988). $X$-AREA. Stoe \& Cie, Darmstadt, Germany.

Ye, J. H., Ponnudurai, R. \& Schaefer, R. (2001). CNS Drug Rev. 7, 199-213. 